\title{
A hybrid RANS model of wing-body junction flow
}

\author{
Aldo Rona $^{a^{*}}$, M.F.F. El-Dosoky ${ }^{b}$, and D.S. Adebayo ${ }^{a, c}$ \\ ${ }^{a}$ Department of Engineering, University of Leicester, LE1 7RH, UK. \\ Tel. +44 116252 2510, Fax. +44 116252 2525. ar45@le.ac.uk; dsa5@le.ac.uk; \\ ${ }^{\mathrm{b}}$ Mechanical Department, Faculty of Engineering, Assiut University, Egypt. mffe1@aun.edu.eg \\ 'School of Engineering, University of Wolverhampton, Telford TF2 9NT, UK, D.Adebayo@wlv.ac.uk
}

\begin{abstract}
The three-dimensional flow separation over the Rood wing-body junction is an exemplar application of separation affecting many important flows in turbomachinery and aerodynamics. Conventional Reynolds Averaged Navier Stokes (RANS) methods struggle to reproduce the complexity of this flow.

In this paper, an unconventional use is made of a hybrid Reynolds Averaged Navier Stokes (RANS) model to tackle this challenge. The hybridization technique combines the Menter $k-\omega-S S T$ model with the one equation sub-grid-scale (SGS) model by Yoshizawa through a blending function, based on the wall-normal distance. The hybrid RANS turbulence closure captured most of the flow features reported in past experiments with reasonable accuracy. The model captured also the small secondary vortex at the corner ahead of the wing nose and at the trailing edge. This feature is scarcely documented in the literature.

The study highlights the importance of the spatial resolution near the wing leading edge, where this localised secondary recirculation was observed by the hybrid RANS model. It also provides evidence on the applicability of the hybrid Menter and Yoshizawa turbulence closure to the wing-body junction flows in aircraft and turbomachines, where the flows are characterised by a substantially time-invariant three-dimensional separation.
\end{abstract}

Keywords: Three-dimensional CFD; hybrid RANS models; Rood wing; horseshoe vortex; flow separation; wing-body junction.

\section{Introduction}

The three-dimensional flow separation that generates a horseshoe vortex is a common occurrence in many practical aerodynamic and hydrodynamic flows. This sort of flows can occur 
when the turbulent boundary layer approaches an obstacle mounted to a surface. This process is encountered in several engineering applications, such as the wing-body junction in aeroplanes, at the blade root in turbomachines, in the convective cooling of electronic components on circuit boards, in rudder-and-keel ship junctions, and in river-bridge flows. The undesirable characteristics associated with the vortical flow, such as the loss in aerodynamic performance, river bed erosion, reduced surface heat transfer, surface hot spots in heat sinks, and vibration and noise generation, attracted the attention of the aerodynamic community towards this problem. Therefore, the horseshoe vortex at these junctions has been intensively studied both numerically and experimentally by Devenport and Simpson [1], Fleming et al. [2], Ölçmen and Simpson [3, 4], Parneix et al. [5], Apsley and Leschziner [6], Jones and Clarke [7], Paciorri et al. [8], Paik et al. [9], Fu et al. [10], Gand et al. [11, 12], Bryan and Klaus [13], and Ryu et al. [14].

These numerical studies highlighted the challenge of reproducing the horseshoe vortex geometry and its associated near-surface flow features, including the separation and reattachment lines, the vortex core trajectory, and the vortex cross-section growth by entrainment, using conventional Reynolds Averaged Navier-Stokes (RANS) simulations, such as the $k-\varepsilon$ and the $k-\omega$ RANS models. Wall-resolved Large Eddy Simulations (LES) can provide greater spatial and temporal resolution than RANS, to resolve the horseshoe vortex flow field. However, the computational effort required for resolving the boundary layers over the horizontal and vertical walls that confine the horseshoe vortex is typically higher in LES than in RANS. As the dominant horseshoe flow structure is mostly steady, this type of flow would lend itself to a steady flow modelling approach in which an appropriate turbulence closure is provided at a lower computational effort than by wall-resolved LES.

This work advances the state-of-art by addressing this challenge using a hybrid RANS model, in which the approach used is somewhat unconventional. The Yoshizawa [15] oneequation model is combined with the $k-\omega$ SST model by Menter [16] to account for the effects of the flow unsteadiness on the time-mean flow away from the walls, while the large-scale vortical structures that form the horseshoe vortex are spatially resolved. The results indicate that this unconventional application of this hybrid turbulence closure gives predictions with a level of detail similar to the ones obtained using full Reynolds stress models or second order moment closures. The simpler algorithm of the Yoshizawa [15] model compared to a full Reynolds stress RANS closure makes this computation more affordable, while attempting to improve on the predictive accuracy of RANS. This makes this unconventional application of the hybrid model of interest to the computational fluid dynamic community engaged in industrial design, where affordable predictions of the time-mean flow are sought around complex geometries.

The fundamental character of horseshoe vortices has been studied on suitably simplified geometries using wings, cubes, and cylinders fastened to the wall surface. A detailed review of the experimental work carried out on these junctions is given by Simpson [17]. The review describes how the interaction between the pressure gradients around the obstacle and the approach turbulent boundary layer generates a three-dimensional separation with horseshoe vortices. This vortical structure is stretched and warped around the appendage, forming an opened necklace 
shape, as shown schematically in Figure 1 . This flow is characterized by subtle features, such as a smaller corner vortex tightly packed around the wing leading edge, which was identified by Fu et al. [10] and confirmed by El-Dosoky [18] and by Lee et al. [19]. The Laser Doppler Velocimetry measurements by Devenport and Simpson [1] showed that the flow around the wing leading edge undergoes non-periodic low frequency velocity fluctuations, leading to a bimodal statistical distribution in Laser Doppler Anemometry measurements. The frequency associated with this unsteadiness is much lower than the frequency of the coherent structures (in the outer layer of the approaching boundary layer) so that its effect on the turbulence structure is small, as suggested by Parneix et al. [5]. The resulting bimodality of the velocity probability density function around the leading edge is likely to affect turbulent quantities. This is because the velocity standard deviation relates to the turbulence intensity, which is higher in a bimodal velocity distribution than in a Gaussian velocity distribution of equal integral area. While this cannot be captured by a conventional two-equation RANS model, its effect on the mean flow parameters is small [5].

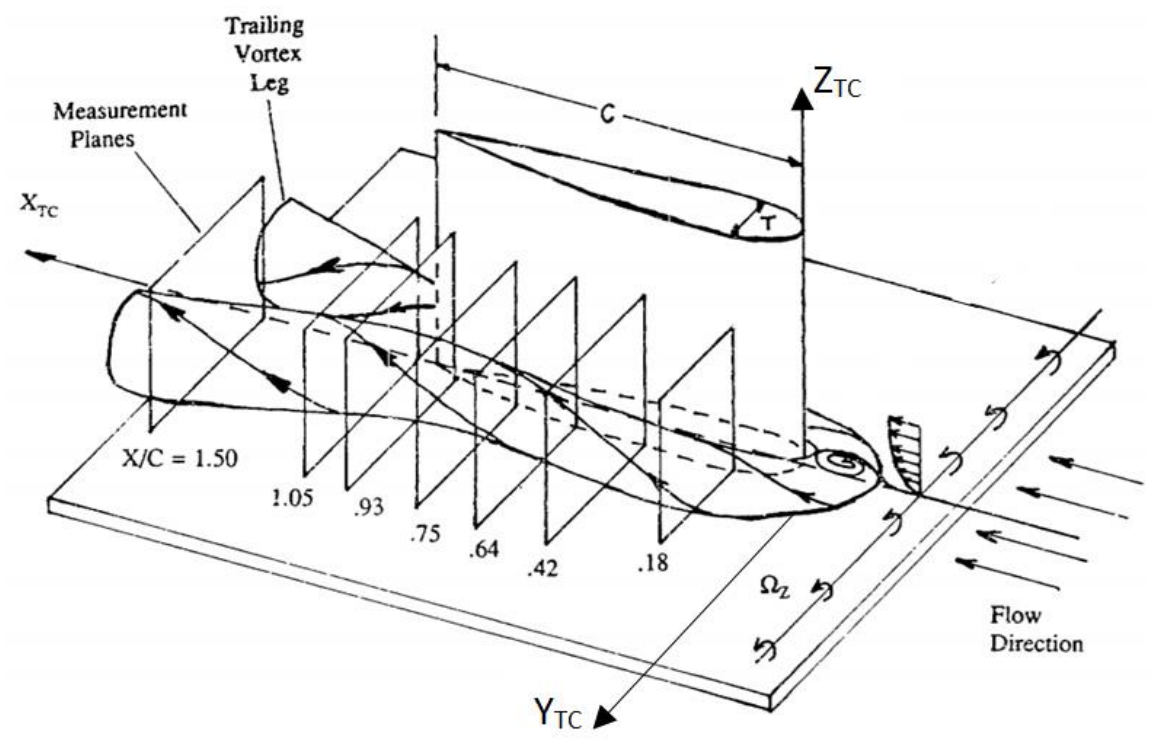

Figure 1. Sketch of the horseshoe vortex around the wing-body junction [2].

Several simulations by authors, including Parneix et al. [5], Apsley and Leschziner [6], Jones and Clarke [7], Paciorri et al. [8], Paik et al. [9], Fu et al. [10], Gand et al. [11], Bryan and Klaus [13], and Ryu et al. [14], studied a 3:2 semi-elliptical nose joined with a NACA0020 tail at the maximum thickness, known as Rood wing, mounted normally on a flat plate with zero angle of attack. Parneix et al. [5] modelled the velocity components and the turbulent kinetic energy upstream of the Rood wing at many locations near the separation line of the horseshoe vortex in the necklace region. They used the V2F turbulence model which extends the standard $k-\varepsilon$ model by incorporating both near-wall turbulence anisotropy and non-local pressure-strain effects, while retaining the linear eddy viscosity assumption. From the comparison with the experimental results, they showed that the $\mathrm{V} 2 \mathrm{~F}$ simulation is able to predict the separation location as well as 
the intensity of the back-flow at the wing upstream symmetry plane more accurately than the standard $k-\varepsilon$ model.

A collaborative university-industry study of a wing-body junction flow is summarized by Apsley and Leschziner [6]. Apsley and Leschziner [6] report numerical simulations obtained using twelve turbulence closure models. They classified these models into three classes as follows: five linear (or isotropic) eddy-viscosity models (different types of $k-\varepsilon, k-\omega$, and $k-g$ models), three non-linear (or anisotropic) eddy-viscosity models (all based on the $k-\varepsilon$ transport equations), and four differential stress (second-moment closure) models. Apsley and Lechziner [6] compared the output from the different turbulence models with experimental measurements, emphasising the structure of the horseshoe vortex and its effects on the forward flow. This study showed that Reynolds stress models offered the most favourable predictive advantages over the other models tested, especially in terms of the far-field structure of the horseshoe vortex, although no model achieved close agreement with the experimental measurements in respect of both mean flow and turbulence quantities.

Another study made by Jones and Clark [7], simulated this flow using the renormalizable $k-\varepsilon$ model, the Reynolds stress model, the V2F model, the Spalart-Allmaras model, and the $k-\omega$ model, running the commercial computational fluid dynamic package ANSYS Fluent. They judged the performance of the turbulence models by comparing the mean velocity components upstream of the wing-body junction as well as the pressure distribution along the wing surface at selected locations. They concluded that, while none of the models tested were able to simulate accurately the correct behaviour of the mean kinetic energy as a function of position, all the turbulence models displayed similarly acceptable levels of accuracy, except the renormalizable $k-\varepsilon$ model. Jones and Clark [7] further confirmed the finding of Parneix et al. [5] that the V2F model gives the closest agreement with the experimental measurements among the five RANS models tested.

Paciorri et al. [8] numerically simulated the wing-body junction using structured and unstructured RANS codes with the one-equation Spalart-Allmaras and the two-equation $k-\varepsilon$ eddy viscosity models. This study carefully assesses the mesh dependence of the predictions by evaluating the grid convergence index and documents the validation of the implemented turbulence models.

Paik et al. [9] followed the Detached-Eddy-Simulation (DES) approach, using the SpalartAllmaras turbulence model for the RANS turbulence closure, to study the bimodality of the velocity probability density function around the horseshoe vortex in a wing-body junction. They stated that there is a discrepancy between simulation and measurements in predicting the coordinates of the horseshoe vortex core. They ascribed this discrepancy to the use of the SpalartAllmaras turbulence model and of the steady inflow conditions. In spite of the poorly predicted vortex core location, the results show the DES approach as a powerful simulation tool for modelling this highly complex turbulent flow. 
Fu et al. [10] modelled by DES a wing body junction flow with the more complex RANS closure of the $k-\omega$ and the $k-\omega-S S T$ models. They controlled the turbulence scale of the Strelets DES method by using Menter's function $\left(F_{2}\right)$ to delay the switching from RANS to LES. They showed that the results from this method deliver the primary horseshoe vortex structure and agree well with the measurements.

A significant element in the predictive ability of the DES is the switching between RANS and LES, for which zonal and non-zonal techniques are available. The zonal technique works by predefining the regions where RANS and LES turbulence closures apply, which is not a convenient approach for modelling unknown flows. The other technique avoids having to predefine regions by automatically choosing the turbulence closure, based on local mesh and flow properties.

The current study uses this latter technique to switch between the Menter [16] and the Yoshizawa [15] turbulence closures. The switch ensures that a RANS Menter [16] layer is always present near a wall and delivers a gradual transition between the Menter [16] and the Yoshizawa [15] turbulence closures by using an appropriate blending function. The presence of a RANS layer over solid walls helps with the proper modelling of attached boundary layers in the current simulation, since, like in a DES, excessive near-wall grid refinement can activate the Yoshizawa [15] model and, like in a DES, it may cause grid-induced flow separation. By distancing the region where the Yoshizawa [15] model is active from the wall, a spurious separation line on the wall is less likely to occur, provided a suitable mesh refinement strategy is used.

Near solid walls, the flow is modelled using the Menter [16] $k-\omega-S S T$, which is a widely used turbulence model. The $k-\omega-S S T$ turbulence closure combines the standard $k-\omega$ model of Wilcox with the Jones-Launder $k-\varepsilon$ model to benefit from the finite value of $\omega$ near the walls and to avoid the strong dependency of the predictions on the values of $k$ and $\omega$ prescribed at the outer boundaries of the computational domain [16]. The model shows a good ability to reproduce the transport of the dominant shear stress in adverse pressure gradient boundarylayers [16]. In this study, the Menter [16] $k-\omega-S S T$ model is coupled with the one equation Sub-Grid-Scale (SGS) model by Yoshizawa [15] that provides the turbulence closure away from the solid boundaries. The details of the numerical approach are stated in the next section.

\section{Hybrid RANS turbulence model}

\subsection{The Menter SST model}

The shear stress transport (SST) model, developed by Menter [16], combines the best qualities of $k-\omega$ and $k-\varepsilon$ models. Menter [16] showed that the SST model exhibits an improved agreement with experiments compared to other classic two-equation RANS turbulence models for a variety of test cases. The SST model gives more accurate predictions in regions of separation in complex flow with a strong adverse pressure gradient [16]. The transport equations for $k$ and $\omega$ are: 


$$
\begin{aligned}
& \frac{D \rho k_{M}}{D t}=\tau_{i j} \frac{\partial u_{i}}{\partial x_{j}}-\beta^{*} \rho \omega k_{M}+\frac{\partial}{\partial x_{j}}\left[\left(\mu+\sigma_{k} \mu_{t, M}\right) \frac{\partial k_{M}}{\partial x_{j}}\right] \\
& \frac{D \rho \omega}{D t}=\frac{\gamma \rho}{\mu_{t, M}} \tau_{i j} \frac{\partial u_{i}}{\partial x_{j}}-\beta \rho \omega^{2}+\frac{\partial}{\partial x_{j}}\left[\left(\mu+\sigma_{\omega} \mu_{t, M}\right) \frac{\partial \omega}{\partial x_{j}}\right]+\underbrace{2 \rho\left[1-F_{1}\right] \sigma_{\omega 2} \frac{1}{\omega} \frac{\partial k_{M}}{\partial x_{j}} \frac{\partial \omega}{\partial x_{j}}}
\end{aligned}
$$

where $\tau_{i j}$ is the turbulent shear stress and is modelled by:

$$
\tau_{i j}=\mu_{t}\left(\frac{\partial u_{i}}{\partial x_{j}}+\frac{\partial u_{j}}{\partial x_{i}}-\frac{2}{3} \frac{\partial u_{k}}{\partial x_{k}} \delta_{i j}\right)-\frac{2}{3} \rho k_{M} \delta_{i j}
$$

The coupling function $F_{1}$ is given by:

$$
\begin{aligned}
& F_{1}=\tanh \left(\arg _{1}{ }^{4}\right), \arg _{1}=\min \left[\max \left(\frac{\sqrt{k_{M}}}{0.09 \omega y} ; \frac{500 \mu}{\rho \omega y^{2}}\right) ; \frac{4 \rho \sigma_{\omega 2} k_{M}}{C D_{k \omega} y^{2}}\right] \\
& C D_{k \omega}=\left[2 \rho \sigma_{\omega 2} \frac{1}{\omega} \frac{\partial k_{M}}{\partial x_{j}} \frac{\partial \omega}{\partial x_{j}} ; 10^{-20}\right]
\end{aligned}
$$

where $y$ is the distance to the nearest wall and $C D_{k \omega}$ represents the positive part of the crossdiffusion term in Equation 2. The constants of the model are obtained by blending the constants in the $k-\omega$ and $k-\varepsilon$ models using the coupling function $\mathrm{F}_{1}$ as follows:

$$
\left[\begin{array}{c}
\sigma_{k} \\
\sigma_{\omega} \\
\beta \\
\gamma
\end{array}\right]=F_{1}\left[\begin{array}{c}
\sigma_{k 1} \\
\sigma_{\omega 1} \\
\beta_{1} \\
\gamma_{1}
\end{array}\right]+\left(1-F_{1}\right)\left[\begin{array}{c}
\sigma_{k 2} \\
\sigma_{\omega 2} \\
\beta_{2} \\
\gamma_{2}
\end{array}\right]
$$

The $k-\omega$ constants are $\sigma_{k 1}=0.85, \sigma_{\omega 1}=0.5, \beta_{1}=0.075, \beta^{*}=0.09, \gamma_{1}=\frac{\beta_{1}}{\beta^{*}}-\frac{\sigma_{\omega 1} \kappa^{2}}{\sqrt{\beta^{*}}}$, and $\kappa=0.41$ while the $k-\varepsilon$ constants are $\sigma_{k 2}=1.0, \sigma_{\omega 2}=0.856, \beta_{2}=0.0828, \beta^{*}=0.09$, $\gamma_{2}=\frac{\beta_{2}}{\beta^{*}}-\frac{\sigma_{\omega 2} \kappa^{2}}{\sqrt{\beta^{*}}}$, and $\kappa=0.41$.

By enforcing the assumption by Bradshaw [20] that the turbulent shear stress in the boundary layer is equal to $\rho \alpha_{1} k_{M}$, Menter's SST turbulent eddy viscosity can be obtained from $\mu_{t, M}=\frac{\rho \alpha_{1} k_{M}}{\max \left[\alpha_{1} \omega ;\left|S_{i j}\right| F_{2}\right]}$, where $\alpha_{1}=0.31, S_{i j}$ is the strain rate tensor $S_{i j}=\frac{1}{2}\left(\frac{\partial u_{i}}{\partial x_{j}}+\frac{\partial u_{j}}{\partial x_{i}}\right)$, and its magnitude $\left|S_{i j}\right|=\sqrt{2 S_{i j} S_{i j}}$.

$$
F_{2}=\tanh \left(\arg _{2}^{4}\right), \arg _{2}=\max \left(2 \frac{\sqrt{k_{M}}}{0.09 \omega y} ; \frac{400 \mu}{\rho \omega y^{2}}\right)
$$


Menter [16] suggested using a limiter $P_{k}$ for the turbulent production term to prevent the unrealistic build-up of eddy viscosity in the stagnation regions. The limiter bounds the production term to 20 times the destruction term and replaces $\tau_{i j} \frac{\partial u_{i}}{\partial x_{j}}$ in the $k$ transport equation.

$$
P_{k}=\min \left[\tau_{i j} \frac{\partial u_{i}}{\partial x_{j}} ; 20 \beta^{*} \rho \omega k_{M}\right]
$$

Menter [16] tested this limiter carefully and reported that this limiter does not change the predicted flow field of well-developed turbulent flows because the maximum level for the ratio of production term to destruction term reaches only up to two inside a shear layer.

\subsection{The Yoshizawa one equation turbulence closure model}

The classic approach to obtaining a large eddy simulation uses the filtered Navier-Stokes equations closed by a one-equation Sub-Grid-Scale (SGS) kinetic energy model. Many applications of SGS models are documented in the literature, including Liu et al. [21], Dahlström and Davidson [22], Davidson and Peng [23], and Orkomi, et al. [24]. Dahlström and Davidson [22] used the one equation SGS model by Yoshizawa [15]. In this model, the SGS transport equation for the kinetic energy $k_{Y}$ is given by

$$
\frac{D \rho k_{Y}}{D t}=\tau_{i j} \frac{\partial u_{i}}{\partial x_{j}}-C_{d} \frac{\rho k_{Y} \frac{2}{3}}{\Delta}+\frac{\partial}{\partial x_{j}}\left[\left(\mu+\sigma_{k} \mu_{t, Y}\right) \frac{\partial k_{Y}}{\partial x_{j}}\right]
$$

where $\tau_{i j}$ is the SGS stress tensor, $\Delta$ is the filter width and is set to the cubic root of the volume of the cell $i\left(\Delta=\sqrt[3]{V_{i}}\right), \mu_{t, Y}=\rho C_{s} \Delta \sqrt{k_{Y}}$, and $\sigma_{k}=1.0$. As in Dahlström and Davidson [22], this filter width definition is linked to the computational mesh, which is designed to suit specific applications, thereby providing a good local control on the filter width. Dahlström and Davidson [22] explore the performance of different filter width definitions in a channel flow simulation, showing that $\Delta=\sqrt[3]{V_{i}}$ performs better than $\Delta=\min \left(V_{i}\right)$.

The Yoshizawa [15] constants $C_{d}$ and $C_{s}$ are problem-dependent and can be evaluated from the corresponding Smagorinsky constant for the wing body junction flow. In the equilibrium conditions, where the production and dissipation rates are in balance, the SGS model recovers the Smagorinsky subgrid eddy viscosity model, so that

$$
\mu_{t, Y}=\rho \sqrt{\frac{C_{s}}{C_{d}}} C_{s} \Delta^{2}\left|S_{i j}\right|=\rho C_{S m a g}^{2} \Delta^{2}\left|S_{i j}\right|
$$

The Smagorinsky constant is related to the Yoshizawa [15] constants by $C_{S m a g}=\left(\frac{C_{s}^{3}}{C_{d}}\right)^{0.25}$ and its value is flow-dependent and it ranges from 0.065 to 0.2 . 
For the flow around bluff bodies and for a complex flow with separation, $C_{S m a g}$ is taken as 0.1 by Rodi [25] and by Jaffŕezic et al. [26]. In this study, $C_{s}$ is calculated using $C_{\text {Smag }}=0.1$ and $C_{d}=1.0$.

\subsection{The hybrid RANS model}

The present hybrid RANS model combines the Menter [16] turbulent kinetic energy equation with the Yoshizawa [15] turbulent kinetic energy equation using a weighting function $\Gamma$. The resulting transport equation for the turbulent kinetic energy $k_{T}$ is

$$
\frac{D \rho k_{T}}{D t}=\tau_{i j} \frac{\partial u_{i}}{\partial x_{j}}-\left(\Gamma \rho \beta^{*} k_{T} \omega+(1-\Gamma) C_{d} \frac{\rho k_{T}^{\frac{3}{2}}}{\Delta}\right)+\frac{\partial}{\partial x_{j}}\left[\left(\mu+\sigma_{k} \mu_{t}\right) \frac{\partial k_{T}}{\partial x_{j}}\right]
$$

The hybrid eddy viscosity is given by

$$
\mu_{t}=\Gamma \mu_{t, M}+(1-\Gamma) \mu_{t, Y}
$$

The blending function $\Gamma$ is defined as $\Gamma=\tanh \left(\xi^{4}\right)$, where $\xi=\max \left[\frac{L_{T}}{y} ; \frac{500 v \beta^{*} L_{T}}{\sqrt{k_{T}} y^{2}}\right]$ and $L_{T}=\frac{\sqrt{k_{T}}}{\beta^{*} \omega}$.

The hybrid technique solves the resulting turbulent kinetic energy equation in combination with the transport equation for $\omega$. Close to the wall, $y \rightarrow 0$ and the blending function approaches unity $(\Gamma \rightarrow 1.0)$, as it can be shown by applying de l'Hopital's theorem to the blending function equation in the $\lim _{y \rightarrow 0} \Gamma$. The Yoshizawa [15] component of the hybrid turbulence closure model becomes less important as $y \rightarrow 0, k_{T}$ represents $k_{M}$, and the RANS solution with the Menter [16] turbulence closure is recovered. Conversely, away from the wall, the blending function approaches zero $(\Gamma \rightarrow 0.0), k_{T}$ represents $k_{Y}$, and the Yoshizawa [15] solution becomes dominant. This behaviour is exemplified in Figure 2, which is derived from the wing-body junction simulations presented in the next section. Figure 2 shows the iso-chromatic levels of the blending function $\Gamma$ in the symmetry plane upstream of the wing body junction leading edge, which is labelled as plane A in Figure 4. Figure 2 shows the Menter [16] closure dominant region near the flat plate and the wing appendage by red and the Yoshizawa [15] closure dominant region by blue. The location of the interface between the $k-\omega \mathrm{SST}$ model and the one-equation $k_{s g s}$ model is shown as the layer enclosed between the dashed and the dash-dot lines, which denote respectively $\Gamma \approx 0.8$ and $\Gamma \approx 0.2$. The dashed line denotes the near-wall interface boundary, which is where the $k-\omega$ SST model starts to blend with the $k_{s g s}$ model but the $k-\omega$ SST model is still driving the value of the specific kinetic energy. The dash-dot line denotes the outer interface boundary, which is where the $k_{s g s}$ model starts to blend with the $k-\omega$ SST model but the $k_{s g s}$ model is still driving the value of the specific kinetic energy. 


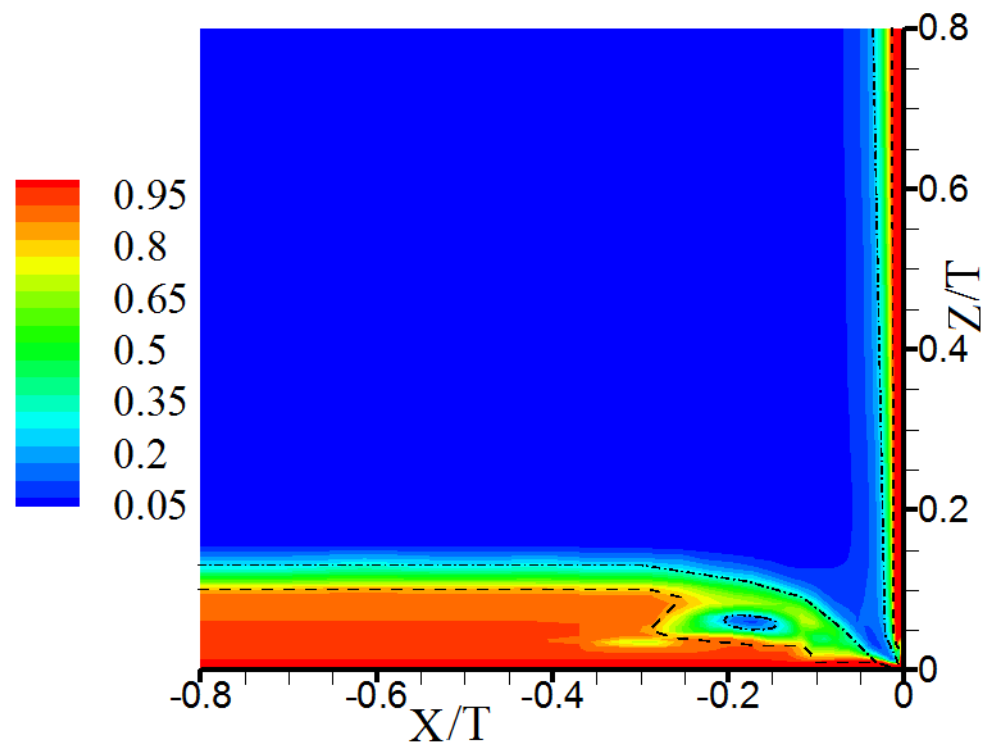

Figure 2. Colour levels of the blending function $\Gamma$ at the symmetry plane $A$ and interface layer between the Menter [16] $k-\omega$ SST and the Yoshizawa [15] $k_{s g s}$ models.

\subsection{RANS model implementation}

The RANS model described in sections 2.1-2.3 has been implemented in the in-house computational fluid dynamics (CFD) software Cosmic. This scheme is an extension of the twodimensional compressible finite-volume scheme by Bennett [27] to three-dimensions. The flow domain is discretized in space using a three-dimensional structured computational mesh, which defines the unit cell finite volume for the application of the conservative laws. Flux Difference Splitting (FDS) is applied on the unit cell control boundaries. The inviscid fluxes are estimated using the second-order implementation of Roe's approximate Riemann solver [28], while viscous fluxes are estimated by the Gauss divergence theorem using a second-order central stencil. Variables at the unit cell interfaces are reconstructed using the Monotone Upwind Scheme for Conservation Laws (MUSCL) by Van Leer [29], which enables up to a third-order accurate spatial reconstruction in regions of smooth flow. To produce a Total Variation Diminishing (TVD) numerical method, the MinMod flux limiter by Roe is applied to calculate the cell interface fluxes. The entropy violation inherent in the Roe scheme is corrected using the formulation by Kermani and Plett [30]. This formulation was made to prevent the occurrence of expansion shocks in the vicinity of a sonic expansion and provides an entropy fix for the Roe scheme. The flow states are updated using a second-order accurate explicit Runge-Kutta time integration, using the low-storage (compact) formulation of $\mathrm{Hu}$ et al. [31]. In the current application, the Runge-Kutta integration is used to update the flow state to numerical convergence and it is not intended to reproduce the evolution of the flow through time. The update at each iteration is constrained to a Courant number of 0.25 , to satisfy the Courant Friedrichs-Lewy (CFL) condition and to preserve the numerical stability of the computations. The computations were run on an eight cores computer cluster managed by the Mathematical Modelling Centre, at the University of Leicester. 


\subsection{Test-case geometry and boundary conditions}

The model consists of a flat plate upon which the Rood wing is fixed, as shown in Figure 1. The wing maximum thickness $(T)$, the chord-to-thickness ratio $(C / T)$, and the Reynolds number based on the wing thickness are $0.0717 \mathrm{~m}, 4.254$, and 115000 respectively. An (X, Y, Z) wind axes type Cartesian reference system is used in Figure 1 , with the origin located at the wing root leading edge.

A computational domain is defined around the wing, as shown in figure 3 . The Rood wing is rendered by the hatched vertical wall in figure 3 , located where indicated by the black arrow. The dimension of the Rood wing is deliberately small compared to the computational domain, so as to prevent numerical blockage by the placement of the computational boundaries in this simulation far from the wing. This test case is symmetric along the X-axis, so the computational covers only half of the physical domain. The computational domain floor at $Z=0$ lies on the flat plate. The computational domain ceiling is set at constant $Z=3 \mathrm{~T}$. The computational domain extends from $X$ $=-18.24 \mathrm{~T}$ to $\mathrm{X}=10 \mathrm{~T}$ and from $\mathrm{Y}=0$ to $\mathrm{Y}=18.24 \mathrm{~T}$.

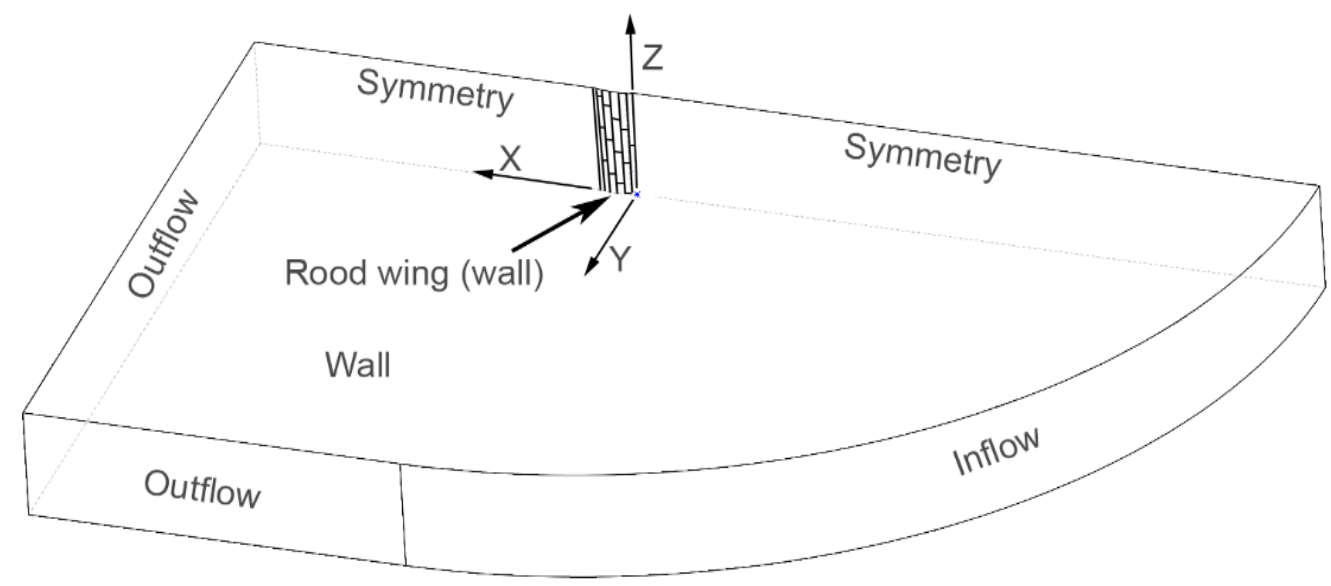

Figure 3. Geometry of the computational domain.

Figure 3 shows the boundary conditions used around the perimeter of the computational domain. A subsonic Inflow boundary condition is imposed between $Z=0$ and $Z=3 \mathrm{~T}$ over the quartercylinder surface between $(X=-18.24 \mathrm{~T}, \mathrm{Y}=0)$ and $(X=0, Y=18.24 \mathrm{~T})$, using the experimental data from the European Research Community on Flow, Turbulence and Combustion (ERCOFTAC, [32]) database. An Outflow boundary condition is applied at $\mathrm{X} / \mathrm{T}=10$ and at $\mathrm{Y} / \mathrm{T}=18.24$, where a zero streamwise pressure gradient is imposed. At $\mathrm{Y} / \mathrm{T}=0$, the symmetry boundary condition is applied. As in Apsley and Leschziner [6], a symmetric boundary condition is set at $Z / T=3$. For all walls, the no-slip adiabatic (Wall) boundary condition is used. The mesh, boundary conditions, and the initial solution file are written in the CGNS format [33], using a bespoke in-house CFD pre-processor written by the authors in Fortran 90. 


\subsection{Computational mesh}

The computational domain is discretised into an assembly of finite-volumes by generating a C-type grid around the wing, as shown in Figure 4 (a). This is then extruded along the wingspan to obtain the three-dimensional computational mesh shown in Figure 4 (b). Numerical mesh stretching is used near physical walls in the flow domain to capture the near-wall flow features. Two incrementally refined, structured multi-block meshes were produced by the bespoke Fortran 90 program. These are referred to as the baseline mesh and the refined mesh. On the baseline mesh, the height of the centre of the first cell closet to the wall is kept within the viscous sublayer $\left(\mathrm{y}^{+}<1.5\right)$, where $\mathrm{y}^{+}$is the cell centre height normalized by the friction velocity and by the air kinematic viscosity. The number of cells in the $X, Y$, and $Z$ directions on the baseline mesh are 112, 50 , and 50 , respectively.
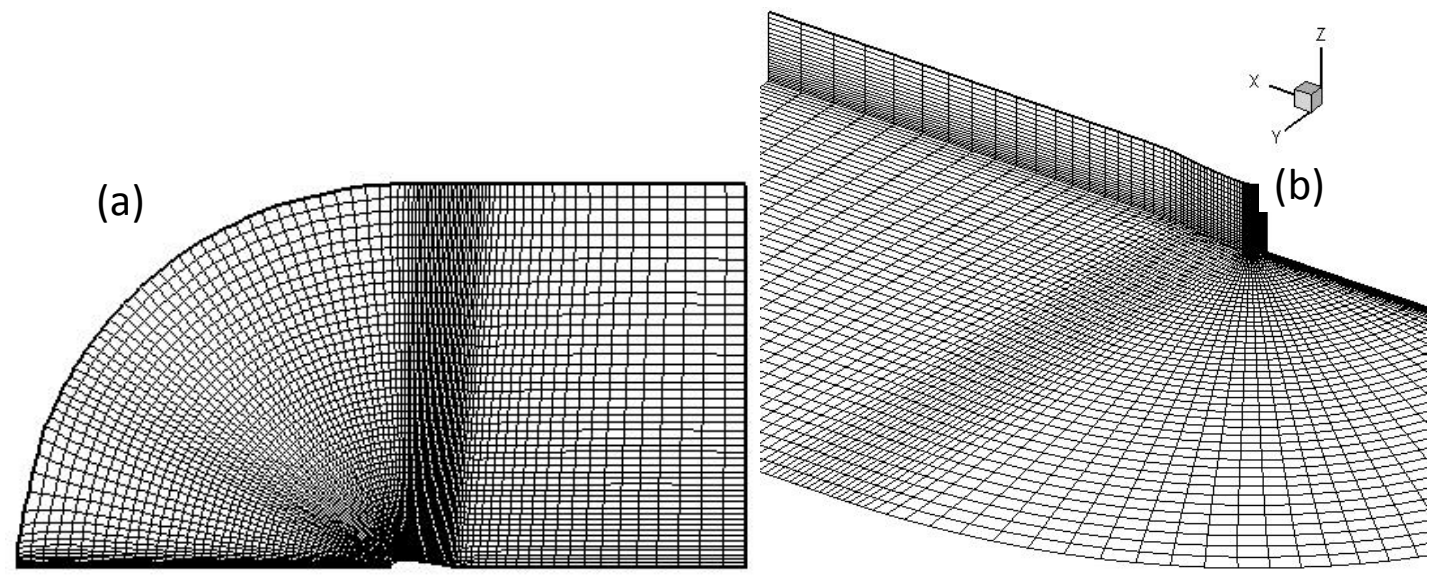

Figure 4. Structured computational mesh: (a) plan view (b) diametric view. For clarity, one every two mesh points in $X, Y$ and $Z$ directions is plotted.

The refined mesh is obtained by increasing the spatial discretization by a factor of 1.5 along the three curvilinear directions. In the refined mesh, the wall-normal distance of the centre of the first cell closest to the wall is less than $0.00047 \mathrm{~T}$ and is designed to give $\mathrm{a}^{+}<0.5$. The number of cells in the $X, Y$, and $Z$ directions on the refined mesh are 168,76 , and 76 respectively. This mesh contains approximately one million cells.

\subsection{Mesh sensitivity analysis}

The sensitivity of the predictions to the level of spatial discretization is considered by comparing the predictions obtained with the baseline mesh to the ones obtained with the refined mesh, on the flat plate, at $Z=0$, and on the symmetry plane $A$ shown in Figure 4 . The inflow freestream velocity is used to normalise the predicted velocity components. 




Figure 4. The upstream symmetry plane $A$ at $X / T=0$, the maximum wing thickness plane $B$ at $X / T=$ 0.18 , and the just downstream the trailing edge plane $\mathrm{C}$ at $\mathrm{X} / \mathrm{C}=1.05$.

Figure 5 shows contours of the static pressure coefficient on the $Z=0$ plane predicted with the baseline mesh (bottom) and with the refined mesh (top). The pressure distribution about the $Y=0$ plane is substantially symmetric, which indicates that the predicted pressure field is substantially unchanged by the increased spatial resolution of the refined mesh.



Figure 5. Contours of static pressure coefficient $C_{p}$ for the baseline and refined meshes.

Figure 6 shows the normalised streamwise and flow-normal velocity components on plane A of Figure 4, predicted with the baseline mesh (dashed lines) and with the refined mesh (solid lines). There is overall a good overlap among the profiles, with small differences among the two predictions observable near the wing nose. Specifically, at $X / T=-0.05$, the refined mesh predicts a lower streamwise velocity minimum than the baseline mesh. Still, both minima are located at approximately $\mathrm{Z} / \mathrm{T}=0.004$. The refined mesh more clearly displays the $\mathrm{U} / \mathrm{U}$ minimum decreasing monotonically its $Z / T$ location with increasing $X / T$ than the baseline mesh, over the range $-0.3<$ $X / T<-0.05$. The $U /$ Uo profile from the baseline mesh at $X / T=-0.25$ displays a small waviness about the corresponding profile from the refined mesh, approximately at $Z / T=0.04$. This feature 
is likely to be a numerical artefact from the spatial interpolation used in the post-processing of the results. The differences notwithstanding, Figures 5 and 6 indicate that there is good overall agreement among the predictions. The authors have elected to use the refined mesh as a prudential approach for obtaining the results presented in the remainder of this paper.
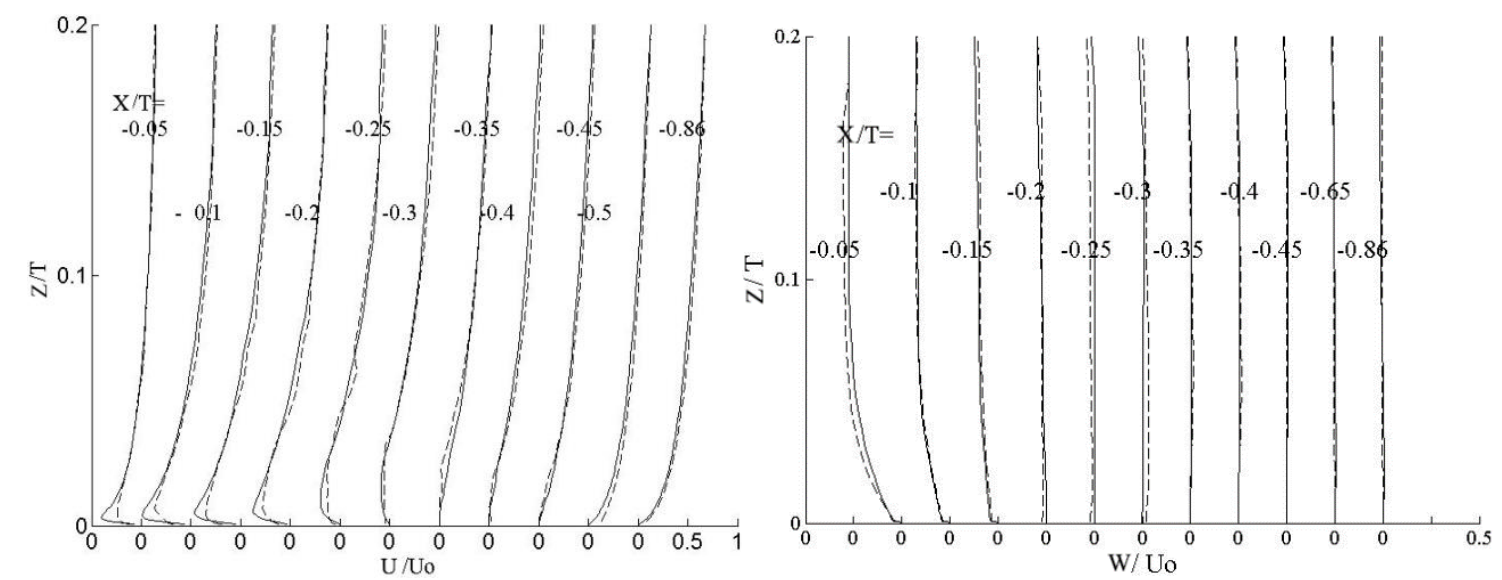

Figure 6. Streamwise and flow-normal velocity profiles for the baseline mesh (----) and the refined mesh (-) at the symmetry plane A defined in Figure 4.

\section{Results and discussion}

\subsection{Symmetry plane upstream of the wing leading edge}

One of the most important aspects in this flow simulation is to predict the horseshoe vortex around the wing-body junction sketched in Figure 1 . To this end, the simulation needs to locate correctly the vortex core in the upstream symmetry plane, corresponding to plane $A$ in Figure 4. In general, RANS simulations with either linear or nonlinear eddy viscosity models are not able to capture the location and the shape of this vortex as well as the increase of the turbulent kinetic energy in the vicinity of the junction [9]. Figures 7 (a) and (c) shows the predicted in-plane streamlines and velocity vectors on the symmetry plane next to the corresponding experimental results, shown in Figures 7 (b) and (d). Both simulation and experiment display a horseshoe vortex core close to the floor. The vortex has an elliptical shape with a clockwise direction of rotation.

The measurements of Devenport and Simpson [1] in Figures 7 (b) and (d) show that the locations of the separation point upstream of the wing nose and the vortex core are at $X / T=-0.35$ and at $X / T=-0.2$ respectively. In Figure 7 (a), the location of the separation point and the centre of the vortex are captured slightly upstream than in experiment, Figure 7 (b), by approximately $5 \%$ and $8 \%$ respectively. This earlier separation is ascribable to the use of the $k-\omega-S S T$ model as the RANS component in the hybrid RANS/LES simulation. It is known that the $k-\omega-S S T$ model is more sensitive to the adverse pressure gradient in boundary-layer flows [16]. A discrepancy in the prediction of the separation point is also found in previous DES results by Paik et al. [9] and by Fu et al. [10] and in predictions from different turbulence closure models by Parneix et al. [5], by 
Apsley and Leschziner [6], and by Jones and Clarke [7]. The 5\% discrepancy in the current prediction indicates that DES is arguably an affordable technique that gives engineering accurate results in a rather complex flow.

The streamlines of Figure 7 (a) show a kink located upstream the separation point. The streamline through this wall-attached layer is bent into a hook shape, indicating an up-well in the predicted velocity field. The same feature is visible in Figure 7 (b) starting from $X / T=-0.48$ and terminating just downstream of the separation point at $X / T=-0.33$. Paik et al. [9] stated that this kink is formed due to the vorticity tail that originates from the vortex core and that stretches upstream, parallel to the flat plate.
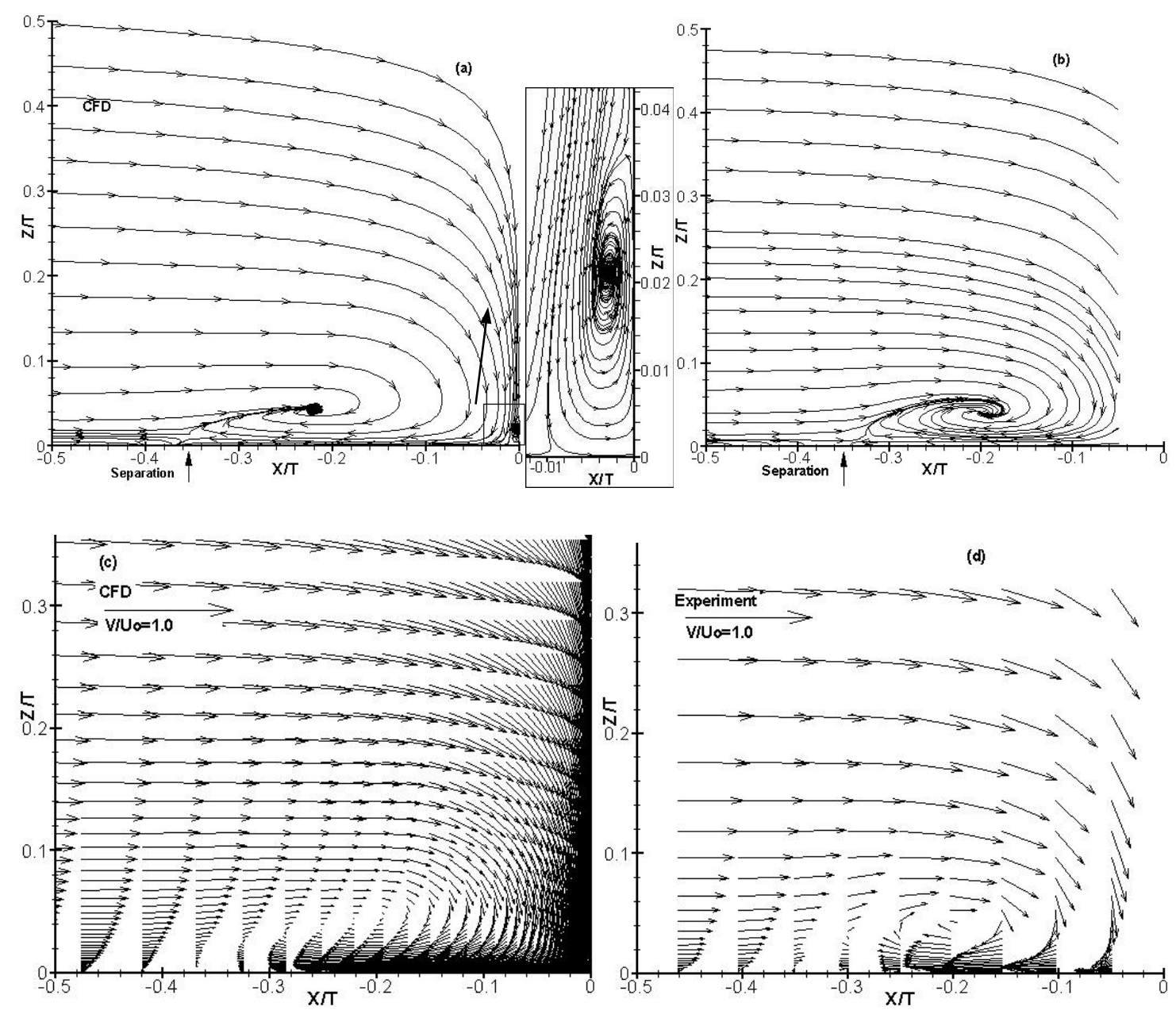

Figure 7. In-plane velocity vectors on the upstream symmetry plane (Plane A) showing the horseshoe vortex core.

In addition to the primary vortex, the simulation showed another secondary vortex close to the junction between the wing leading edge and the flat plate. This feature is shown in the figure inset between Figure 7 (a) and Figure 7 (b). Although the secondary vortex is not clearly captured by Devenport and Simpson [1] through their oil flow visualizations, they commented that 
there is a small region of secondary separation in the corner between the wall and the wing [1]. This secondary separation qualitatively similar to the one predicted at the Rood wing leading edge by three different turbulence models in Fu et al. [10] and in the numerical simulations by Lee et al. [19]. As such, the current hybrid RANS method provides supportive evidence on the location of this secondary recirculation, which is otherwise difficult to detail by experiment, highlighting the useful complementarity between numerical and experimental investigations in complex threedimensional flows.

Figure 8 shows the CFD contour lines of the velocity components (U/Uo, W/Uo) compared to the corresponding experimental contours.
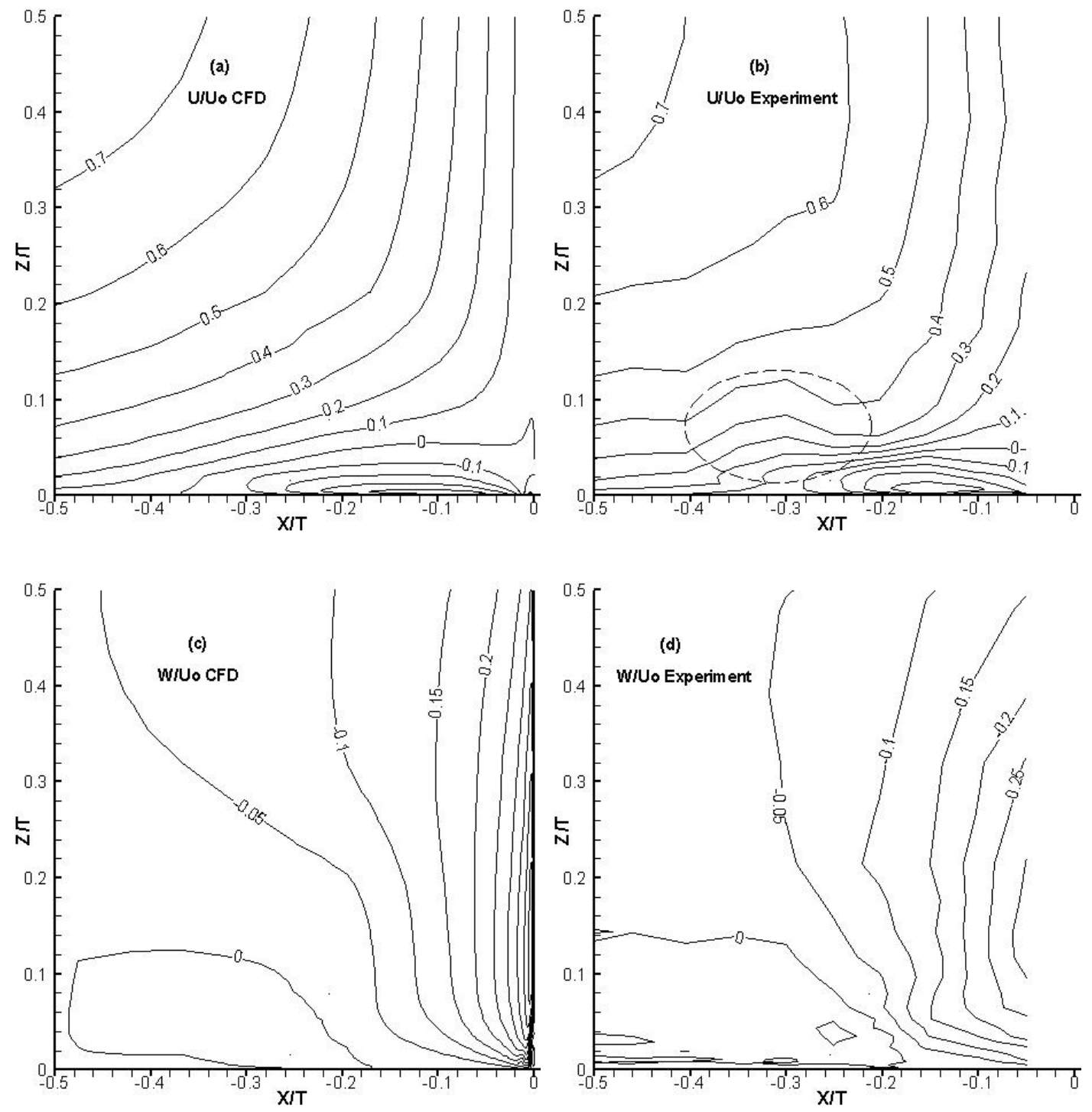

Figure 8. Streamwise and flow-normal velocity distributions in the symmetry plane A.

Figure 8 shows a good quantitative and qualitative agreement between simulation and experiment. The most noticeable difference between the computed contour lines and the 
experimental measurements of the streamwise velocity lies between the separation point and the core of the horseshoe vortex near the wall, where indicated by the dashed line in Figure 8 (b). This difference may be attributed to the calculation of the turbulent eddy viscosity using a sensitive blending function along the separating boundary layer upstream the horseshoe vortex. Specifically, as the flow separates from the flat plate at $\mathrm{X} / \mathrm{T}=-0.35$, the upwell develops under an adverse pressure gradient that promotes the production of turbulent kinetic energy in the DES model.

The turbulent kinetic energy contours at the symmetry plane A of Figure 4 are shown in Figure 9. The computed turbulent kinetic energy contours are compared against the corresponding experimental data. These results confirm that hybrid RANS can better capture an increase in kinetic energy near a corner, like near the wing junction, compared to RANS, which is unable to capture such feature [9].

While the hybrid RANS model has improved the agreement with the measurements, there are still noticeable differences. The mean velocity in the hybrid RANS is modelled by the $k-\omega-S S T$ RANS technique. This model is unable to reproduce the local turbulence anisotropy, leading to an underestimate in the predicted turbulent kinetic energy. Also, streamline curvature generates additional strain rates that significantly affect the turbulent stress production.

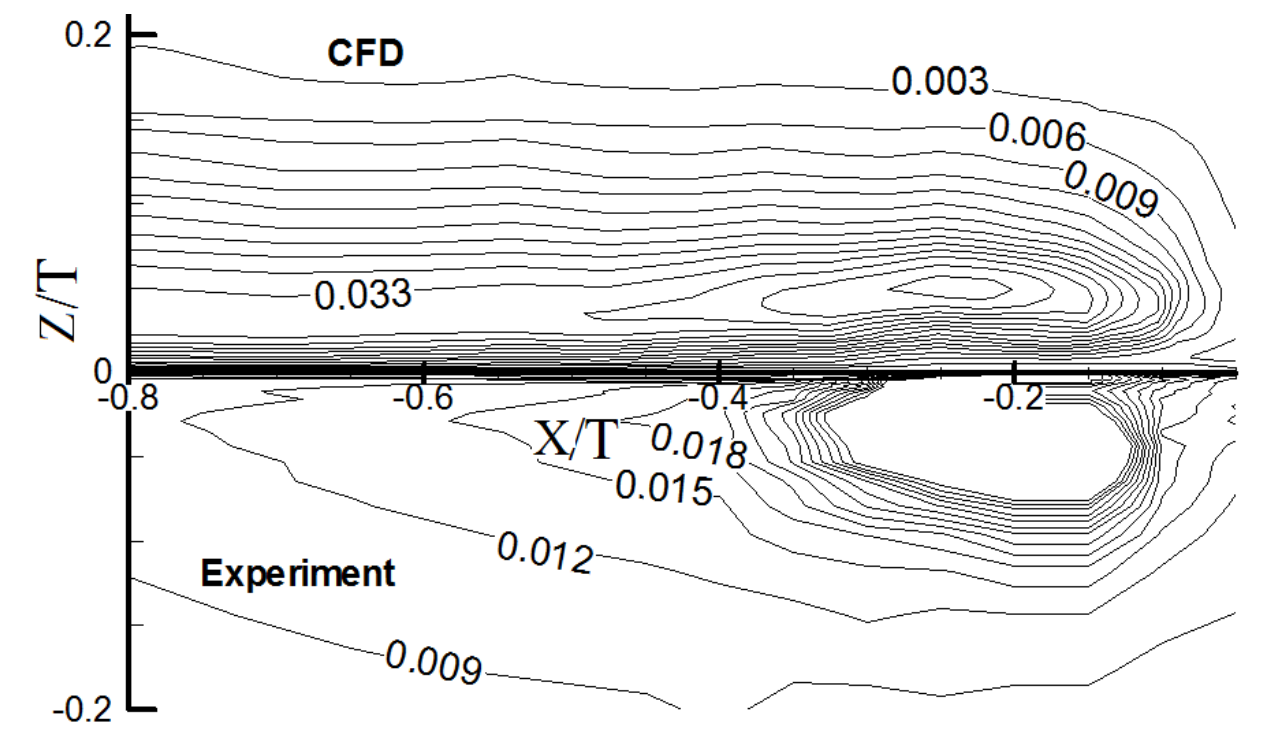

Figure 9. Turbulent kinetic energy distribution in the upstream symmetry plane A, $\Delta k=0.003 U_{0}^{2}$.

\subsection{The wing root flow}

The computed contours of the time averaged pressure coefficient $C_{p}$ on the bottom wall $(\mathrm{Z} / \mathrm{T}=0.0)$ at the wing junction are plotted and compared with the experimental values in Figure 10. Figure 10 shows that the predicted static pressure distribution is in good agreement with the measured data. The pressure rise on approach to the leading edge stagnation point at $(X / T, Y / T)=$ 
$(0,0)$ is reproduced well by the CFD and the same level contours are pretty close to one another on the $\mathrm{Y} / \mathrm{T}=0$ symmetry plane. The location and magnitude of the pressure minimum on the wing surface are also close. This shows that the simulation gives a good agreement of the mainly twodimensional pressure field generated by the wing, aside from secondary flow effects. In general, the pressure along the wing is weakly dependent on the turbulence model, as reported by Apsley and Leschziner [6], and is not greatly affected by the horseshoe vortex, as pointed out by Paciorri et al. [8].



Figure 10. Static pressure coefficient distributions about the wing at $\mathrm{Z} / \mathrm{T}=0$.

The pressure coefficient distribution along the wing surface is tested at two other locations above the flat plate, at $Z / T=0.133$ and at $Z / T=1.726$. The plots of the pressure coefficient at these two locations are shown in Figure 11.
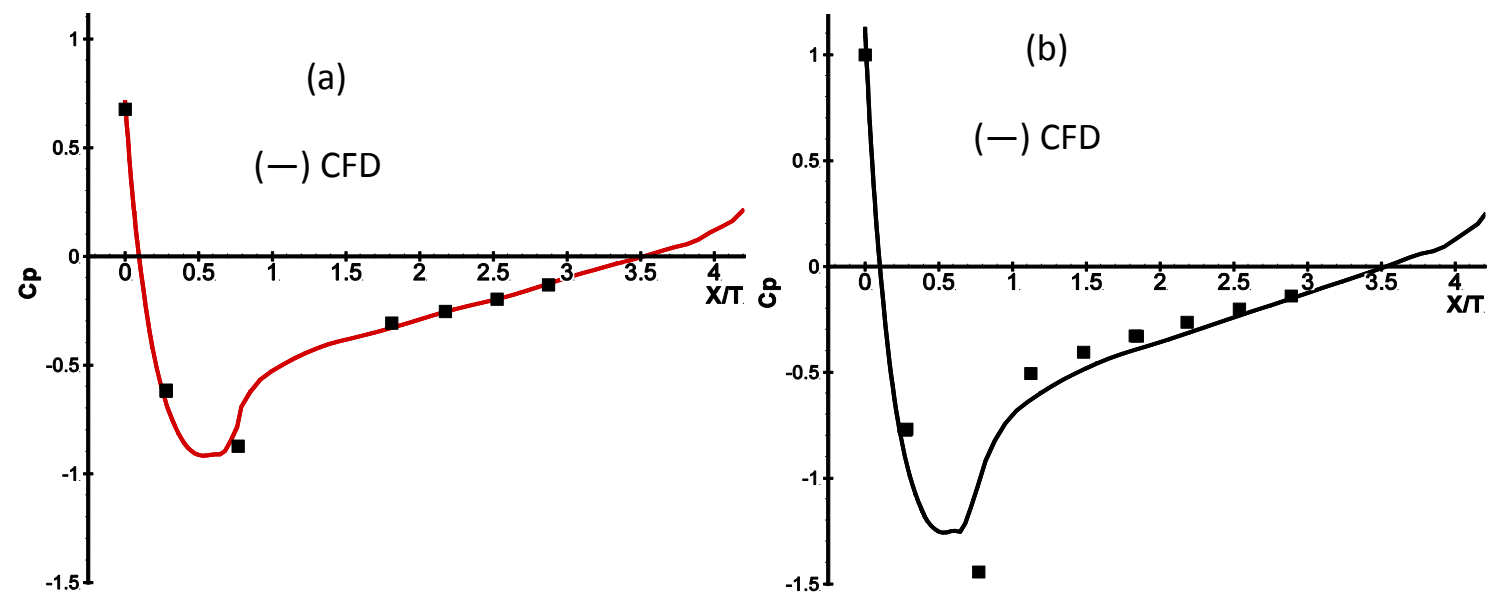

Figure 11. Static pressure coefficient distribution at (a) $\mathrm{Z} / \mathrm{T}=0.133$, (b) $\mathrm{Z} / \mathrm{T}=1.726$.

At $\mathrm{Z} / \mathrm{T}=0.133$, the numerical results are in a good quantitative and qualitative agreement with the 
experimental data while at $\mathrm{Z} / \mathrm{T}=1.726$, the numerical model under-estimates the $\mathrm{Cp}$ minimum. This difference at high $\mathrm{Z} / \mathrm{T}$ may be partly attributed to the difference between the top boundary condition used in experiment and in the simulation. While there is a gap between the top of the wing and the tunnel ceiling wall in the experiment, the simulation used a slip wall as a side fence at the wing tip. This slip wall is modelled as a symmetry boundary as described in section 2.5 . The level of agreement between predicted and measured pressure coefficients on these axial planes is broadly similar to that reported in previous RANS simulations $[7,19]$. There is no appreciable improvement in agreement by using the hybrid RANS method for predicting the $\mathrm{Cp}$ distributions on these planes. Still, the current predictions are obtained using a numerical mesh of one million cells compared to 4.7 million cells in Lee et al. [19] and five million cells in Jones and Clarke [7], indicating a potential reduction in computational cost from using the hybrid RANS model.

Figure 12 shows the predicted streamlines over the flat plate at the wing root compared with the oil flow visualization performed by Ölçmen and Simpson [3]. The flow over the flat plate, which is the body of the wing-body junction geometry, features a three-dimensional separation. Figure 12 shows the stagnation saddle point at the plane of symmetry and the separation line that emanates from this point and warps around the wing. The separation line around the wing represents the path of the horseshoe vortex. A good agreement is noticed between the simulation results and the experimental flow visualization.

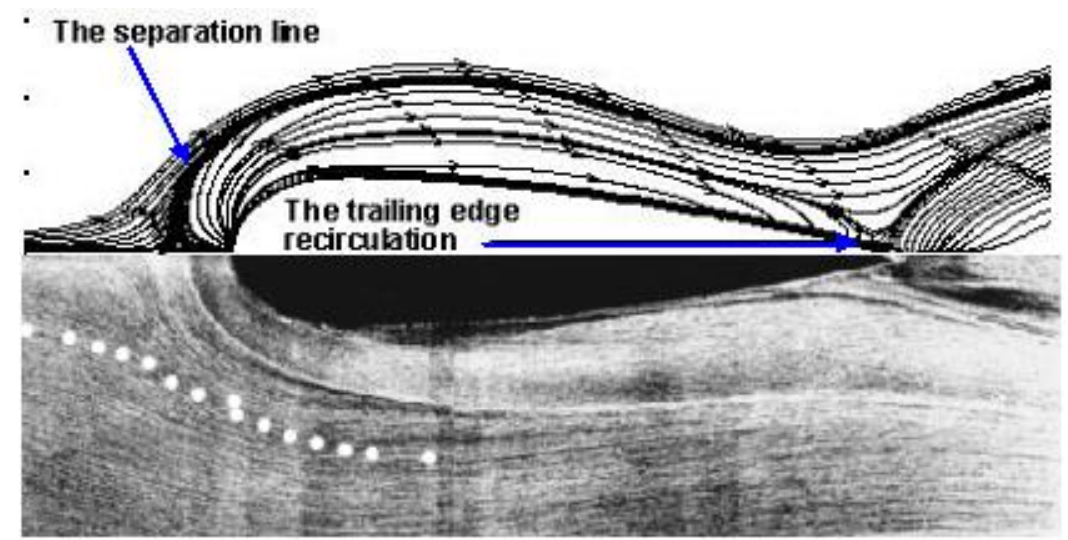

Figure 12. Predicted streamlines against the oil flow visualization by Ölçmen and Simpson [3].

Figure 13 shows the predicted surface streamlines both on the flat plate and on the wing surface from roughly a three-quarters view angle. This figure also includes the surface streamlines on the symmetry plane A as defined in Figure 4. The streamlines over the wing surface indicate that, away from the wing root, the flow remains attached from the leading edge to about $85 \%$ chord. Downstream of $80 \%$ chord, the flow separates from the wing surface, forming the near vertical separation line at about $85 \%$ chord, as shown in Figure 13. Close to the wing root, the flow becomes more three-dimensional and this separation streamline spirals into a vortex. This vortex drives a small recirculation on the flat plate between $90 \%$ chord and the trailing edge. The oil flow visualization of Figure 12 shows a single grey line departing form the wing surface at $80 \%$ chord. This line is evidence of the predicted trailing edge corner recirculation, which is a feature previously identified by Parneix et al. [5]. By combining surface streamlines on the flat plate and 
on the wing surface, the present study has added to the state of art by showing the wing surface vortex that drives the secondary recirculation near the trailing edge observed in experiment.

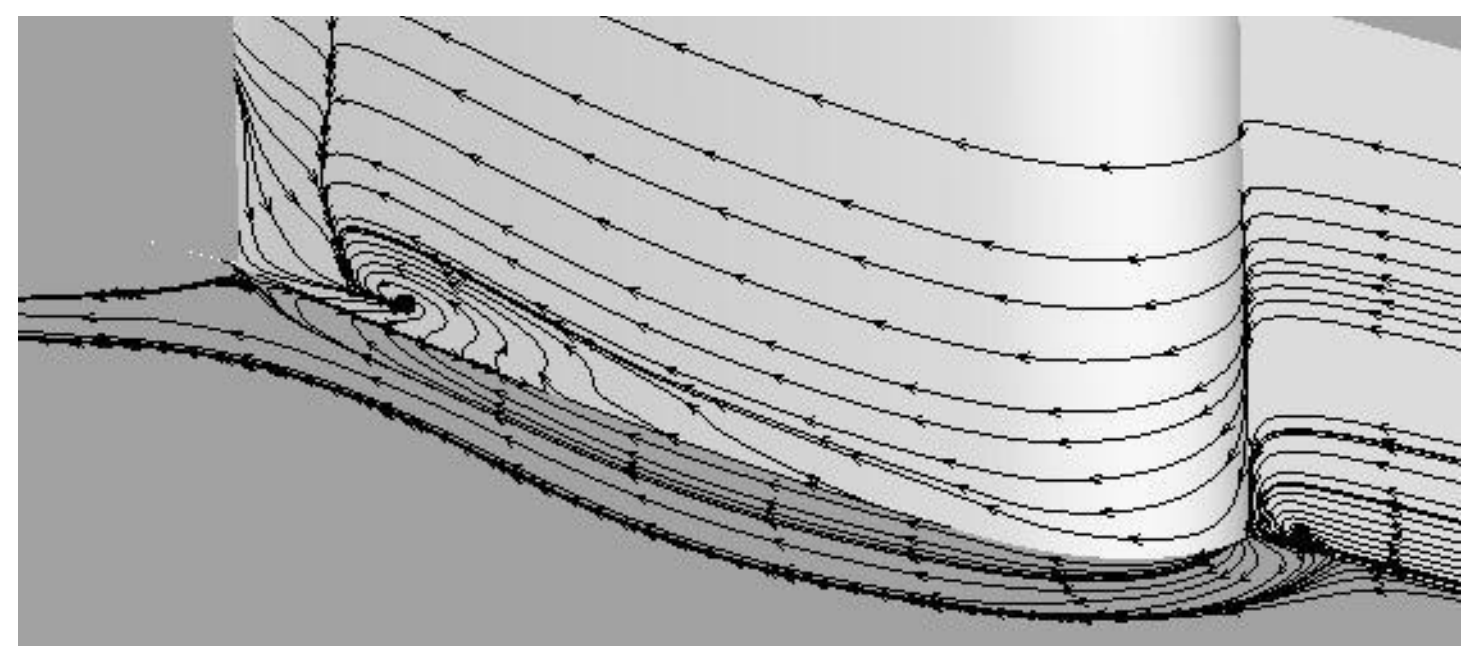

Figure 13. The circulation and trailing edge vortex.

Figure 14 shows the contour plot of the velocity components at the maximum wing thickness compared to the experimental data. The computed streamwise velocity component (U/Uo) matches reasonably well with the experimental data even near the wall and along the wing span. Away from the wing surface, the streamwise velocity is weakly affected by the potential pressure field of the wing and asymptotes to the velocity distribution of two-dimensional boundary layers. Specifically, the $U$ velocity is very low close to the flat plate, at $Z / T=0$, and increases monotonically in the positive $\mathrm{Z} / \mathrm{T}$ direction.

Approaching the wing surface, which is located at $\mathrm{Y} / \mathrm{T}=0.5$, the $\mathrm{U}$ velocity increases due to the wing thickness effect that induces a maximum velocity close to the location of maximum wing thickness. This shows as the location of minimum surface pressure in Figure 10. The streamwise velocity close to the wing surface is highest away from the flat plate, where it is less affected by the skin friction along this surface. A close inspection of Figure 14 (a) shows densely packed $U$ contours close to the wing surface, at $\mathrm{Y} / \mathrm{T}=0.5$. These are due to the growing boundary layer on the wing surface. This boundary layer grows under a favourable pressure gradient from the wing leading edge to plane (B), leading to a thin boundary layer. This effect is not captured by Figure 14 (b), probably due to the limitation in the experiment in measuring the flow velocity close to the wall. This is a common occurrence in LDV data that is affected by surface reflection of the low laser beams on the solid walls.

The contours of the spanwise velocity component $V$ in Figure 14 (c) are in reasonable agreement with the experimental contours in Figure 14 (d), except for a small difference to the side of the wing surface. The $\mathrm{V}$ contours show a horizontally elongated region of relatively high spanwise velocity above the flat plate at $\mathrm{Z} / \mathrm{T}=0.025$. This area of increased spanwise velocity is induced by the horseshoe vortex above the flat plate. Specifically, the anti-clockwise circulation induced by the horseshoe vortex drives the spanwise motion of fluid below the vortex core along 
the flat plate and away from the wing. The proximity of the flat plate increases the magnitude of the spanwise velocity induced by the vortex that adds to the spanwise velocity induced by the wing thickness effect. The near-wall peak is due to a mechanism similar to that of a wing in ground effect, in which the packing of the streamlines due to the non-permeable wall is equivalent to having an image vortex below the wall, driving the spanwise flow.
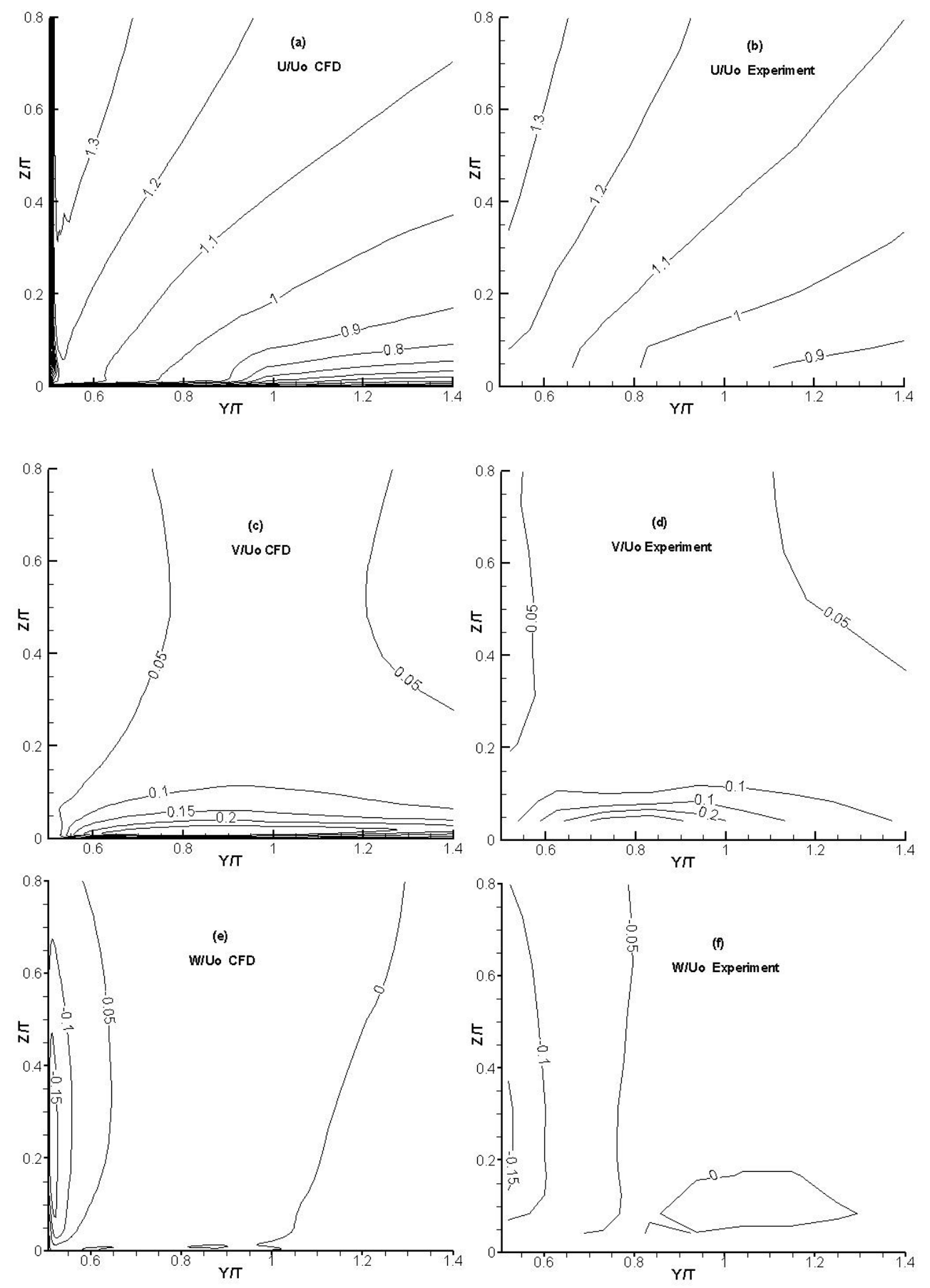

Figure 14. Contours of normalized velocity components at the wing maximum thickness, plane B viewed from downstream. 
The CFD predictions of Figure 14 (c) show that the area of relatively high $\mathrm{V}$ is detached from the flat plate surface and that the near-surface spanwise velocity rapidly decreases to zero at the surface, thus satisfying the non-slip boundary condition at the wall of $V=0$. This near-wall decay is not shown in experiment where it was not possible to measure the velocity in close proximity to the wall. Therefore the current CFD predictions are a good complement to the measurements, displaying understandable flow physics in a region where measurements are difficult to obtain and contributing towards obtaining a full picture of the wing-body junction flow.

Figures 14 (e) and 14 (f) show the predicted and the experimental flow-normal velocity on plane (B). The flow-normal velocity shows a similar trend to that of the spanwise velocity, in that an area of greater flow-normal velocity magnitude is shown close to the wing surface. The extent of this area is smaller compared to that of Figures 14 (c) and 14 (d). The reason for the lower flownormal velocity magnitude close to the wing as compared to the spanwise velocity maximum above the flat plate is that the horseshoe vortex core is farther away from the wing than from the flat plate on plane (B). The agreement between the experiment and computation on plane $B$ in flow-normal velocity is coarser than the one for the other two velocity components, which probably results from the flow-normal velocity being the smallest of the three components, hence suffering from a proportionally greater uncertainty in the measurements [8].

\subsection{Downstream of the trailing edge}

The development of the vortical flow downstream of the trailing edge and in the wake is as important as the flow at the symmetry plane. Figure 15 shows contour plots of the predicted normalized velocity components just downstream of the trailing edge $(X / C=1.05)$ compared to the experimental data. The location of this plane is sketched in Figure 4 as plane C. Figure 15 shows that the simulation gives a fair agreement with the experimental data.

The simulation captured the growth of the thin shear layer separating from the wing trailing edge. This is shown by the streamwise velocity contours packed on the left of Figure 15(a), along the $Y / T=0$ axis. Above the flat plate wall, which in Figure 15 coincides with the $Z / T=0$ axis, the streamwise velocity contours are spread further apart to each other than normal to the $\mathrm{Y} / \mathrm{T}=0$ axis, marking the presence of a thicker boundary layer on the flat plate as compared to the separating shear layer at the wing trailing edge. The presence of the horseshoe vortex is shown by the $S$ shaping of the streamwise velocity contour levels $U / U_{0}=0.7$ and $U / U_{0}=0.8$. The anticlockwise horseshoe vortex generates an upwash-downwash pair that convects high-speed freestream flow into the boundary layer on the downwash side and rises lower speed flow away from the wall on the upwash side. This displaces the streamlines at $Y / T<0.7$ towards the $Z / T=0$ floor and rises the streamlines at $Y / T>0.8$ away from the $Z / T=0$ floor in Figures 14 (a) and 14 (b).

The effect of the anti-clockwise vortex is also visible in the flow-normal velocity contours and in the spanwise velocity contours of Figures 15 (c, d) and $15(e, f)$, respectively. The nearhorizontal contours of Figure 14 (c) above $Z / T=0$ are replaced by a near-wall local maximum at $\mathrm{Y} / \mathrm{T} \approx 0.6$ in Figures 15 (c) and $15(\mathrm{~d})$, with the contour lines bending towards the wall at $\mathrm{Y} / \mathrm{T}>0.6$. 
This pattern can be explained by the anti-clockwise horseshoe vortex centred right above the local maximum at $Y / T \approx 0.6$ sweeping fluid across the flat plate in the $Y$ direction.
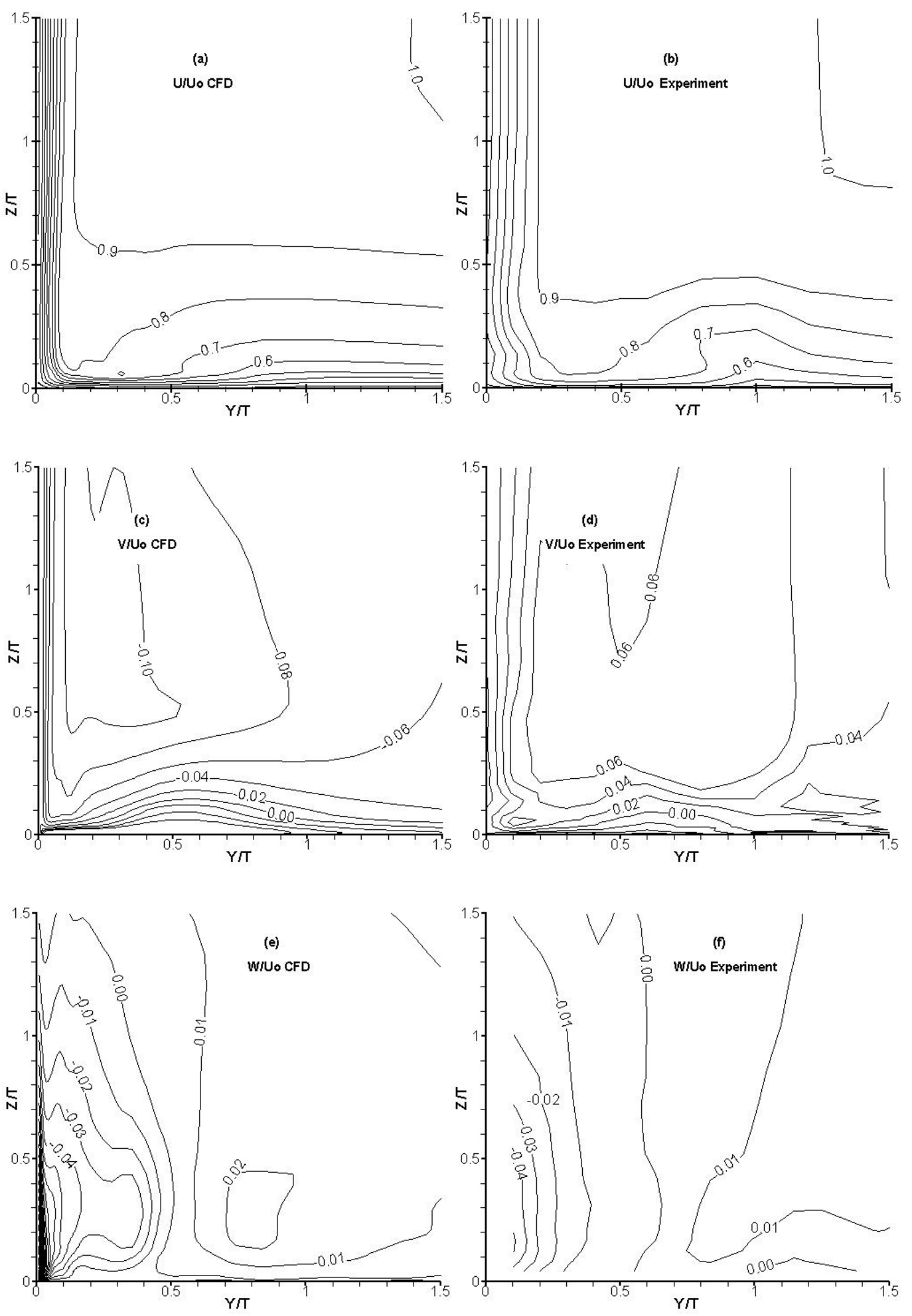

Figure 15. Contours of normalized velocity components at the wing trailing edge, plane $C$ viewed from downstream. 
The near-wall local $Y$ velocity maximum is obtained as the horseshoe vortex induced flow is forced through the narrowest space between the flat plate and the vortex core. The upwash and downwash near the wall form two regions of opposite signed wall-normal velocity, either side of $\mathrm{Y} / \mathrm{T} \approx 0.5$, as shown in Figures 15 (e) and 15 (f). Local regions of low and high wall-normal velocity centred at $\mathrm{Z} / \mathrm{T} \approx 0.3$ identify respectively the downwash peak and upwash peak from the horseshoe vortex on plane $C$. The quantitative comparison of the velocity cross-flow components is difficult because they have small values and, consequently, the uncertainties associated to their measured values become significant [8].

Figure 16 shows the velocity vector field further downstream from the trailing edge, at $X / T$ $=6.38$. Since there is no comparative experimental data in the ERCOFTAC [32] database at this location, the simulation results are compared with the numerical predictions by Apsley and Leschziner [6] obtained by using the Reynolds stress model of Jakirlić and Hanjalić [34]. Figure 16 shows that the hybrid RANS/LES method predicts the vortical flow with approximately the same core centre position and a stronger intensity of the transverse vortex motion with respect to the predictions of Apsley and Leschziner [6].

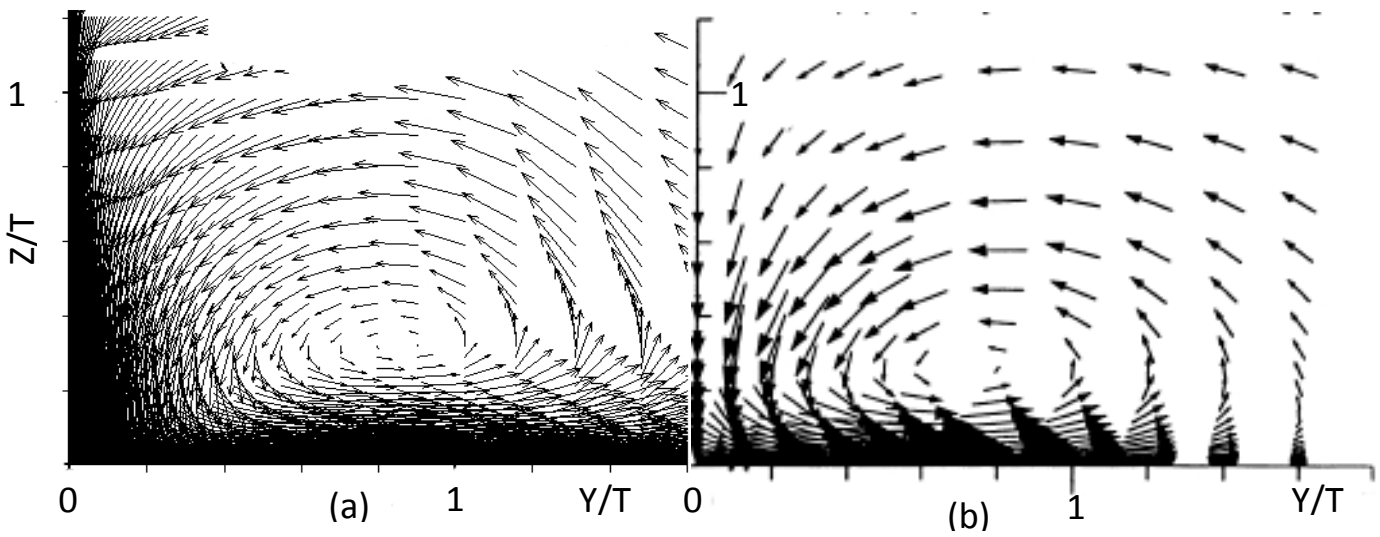

Figure 16. Cross-flow velocity vector maps detailing the horseshoe vortex at $X / T=6.38$, viewed from downstream. (a) Current DES model and (b) Apsley and Leschziner [6].



Figure 17. The horseshoe vortex development downstream of the trailing edge. 
An overall view for the vortical flow downstream the wing is shown in Figure 17. This figure shows the development of the horseshoe vortex and the lifting up of its core in the wake region.

\section{Conclusion}

In the present study, a numerical simulation of the turbulent flow past the wing-body junction has been performed using a hybrid RANS turbulence closure approach. Comparisons between the computed flow field and corresponding experimental data from the ERCOFTAC [32] database are presented. The results show that the hybrid RANS/LES turbulence closure is able to capture salient characteristics of this complex flow with reasonable accuracy. The simulation captured the upwind vortex, the 3-D stagnation saddle point, and the separation line at the symmetry plane, the trailing edge recirculating flow as well as the development of the horseshoe vortex downstream of the trailing edge.

The main finding in this simulation is the ability of the hybrid RANS turbulence closure to capture small vortices which, to some degree, are not captured clearly by the flow visualization techniques and have received to date limited coverage by other simulation methods. The hybrid RANS turbulence closure is able to capture the small secondary vortex in the front of the appendage junction with the flat plate and the trailing edge vortex.

The numerical predictions in this work have clarified interesting aspects of the secondary flow physics in a Rood wing-body junction and have helped to achieve a better understanding and interpretation of the flow physics with respect to the available experimental data. This work has also shown how a hybrid RANS turbulence closure can be used in a non-conventional way to predict complex three-dimensional flow with important steady vortex structures. The lower computational cost of the hybrid approach compared to a full Reynolds stress model makes this technique attractive for industrial design computational fluid dynamics, provided supportive

validation elements are available alongside the CFD predictions to keep this unconventional use of the LES turbulence closure in check against real flow data.

This study has also exposed some limitations of the hybrid RANS turbulence closure. Specifically, the location of the vortex core after the wing trailing edge is predicted slightly upstream of the experimental value and the minimum $\mathrm{Cp}$ at $\mathrm{Z} / \mathrm{T}=1.726$ is under-predicted by the model. Future work can explore whether this difference can be reduced by using an adjustable blending function to better control the extent of the region where the $k-\omega-S S T$ model is active. The other factor which can be altered is the equivalent Smagorinsky constant used to calculate both the eddy viscosity model constant and the destruction rate model constant in the transport equation of the turbulent kinetic energy. Still, the results show that the hybrid RANS turbulence closure is a powerful tool for the simulation of complex three-dimensional flows. 


\section{References}

[1] W.J. Devenport and R.L. Simpson, Time-dependent and time-averaged turbulence structure near the nose of a wing-body junction, Journal of Fluid Mechanics 210 (1990), 23-55. doi: doi.org/10.1017/S0022112090001215.

[2] J.L. Fleming, R.L. Simpson, J.E. Cowling, and W.J. Devenport, An experimental study of a turbulent wing-body junction and wake flow, Experiments in Fluids 14 (1993) 366-378. doi: doi.org/10.1007/BF00189496.

[3] S.M. Ölçmen and R.L. Simpson, An experimental study of a three-dimensional pressure-driven turbulent boundary layer, Journal of Fluid Mechanics 290 (1995), 225-262. doi: doi.org/10.1017/S0022112095002497.

[4] S.M. Ölçmen and R.L. Simpson, Some feature of a turbulent wing-body junction vortical flow, International Journal of Heat and Fluid Flow 27(6) (2006) 980-993. doi: 10.1016/j.ijheatfluidflow.2006.02.019.

[5] S. Parneix, P.A. Durbin, and M. Behnia, Computation of 3-D turbulent layers using the V2F model, Flow, Turbulence and Combustion 60(1) (1998) 19-46. doi: doi.org/10.1023/A:1009986925097.

[6] D.D. Apsley and M.A. Leschziner, Investigation of advanced turbulence models for the flow in a generic wing-body junction, Flow, Turbulence and Combustion 67 (2001) 25-55. doi: doi.org/10.1023/A:1013598401276.

[7] D.A. Jones and D.B. Clarke, Simulation of a wing-body junction experiment using the fluent code (Defence Science and Technology Organisation Report No. DSTO-TR-1731) (2005). Victoria: DSTO Platforms Sciences Laboratory.

[8] R. Paciorri, A. Bonfiglioli, A. Di Mascio, and B. Favini, RANS simulations of a junction flow, International Journal of Computational Fluid Dynamics 19 (2005) 179-189. doi: doi.org/10.1080/10618560410001729126.

[9] J. Paik, C. Escauriaza, and F. Sotiropoulos, On the bimodal dynamics of the turbulent horseshoe vortex system in a wing-body junction, Physics of Fluids 19(4) (2007). doi: doi.org/10.1063/1.2716813. 
[10] S. Fu, Z. Xiao, H. Chen, Y. Zhang, and J. Huang, Simulation of wing-body junction flows with hybrid RANS/LES methods, International Journal of Heat and Fluid Flow, 28 (2007) 1379-1390. doi: doi.org/10.1016/j.ijheatfluidflow.2007.05.007

[11] F. Gand, V. Brunet, and S. Deck, A combined experimental, RANS and LES investigation of a wing-body junction flow. Paper presented at the 40th Fluid Dynamics Conference and Exhibit, Chicago, IL (2010, June).

[12] F. Gand, V. Brunet, and S. Deck, Experimental and numerical investigation of a wing-body junction flow, AIAA Journal, 50(12) (2012) 2711-2719. doi: doi.org/10.2514/1.J051462.

[13] C.H. Bryan and A.H. Klaus, Parametric exploration of wing-body junction flow using computational fluid dynamics, Journal of Aircraft 52(5) (2015) 1492-1509. doi: doi.org/10.2514/1.C032985

[14] S. Ryu, M. Emory, G. laccarino, A. Campos, and K. Duraisamy, Large-eddy simulation of a wing-body junction flow, AIAA Journal, 54(3) (2016) 793-804. doi: doi.org/10.2514/1.J054212.

[15] A. Yoshizawa, Statistical theory for compressible shear flows with the application of subgrid modelling, Physics of Fluids A 29 (1986) 2152-2163. doi: doi.org/10.1063/1.865552.

[16] F.R. Menter, Improved two-equation $k-\omega$ turbulence models for aerodynamic flows (NASA Technical Memorandum No. NASA-TM-103975), Washington, DC: National Aeronautics and Space Administration (1992).

[17] R.L. Simpson, Junction flows, Annual Review of Fluid Mechanics 33 (2001) 415-443. doi: doi.org/10.1146/annurev.fluid.33.1.415.

[18] M.F.F. El-Dosoky, Analytical and CFD methods investigating shroud tip leakage, PhD thesis, University of Leicester, April 2009.

[19] J.P. Lee, J.-H. Chen, C.-Y. Hsin, Study of junction flow structures with different turbulence models, Journal of Marine Science and Technology, 25(2) (2017) 178-185. doi: 10.6119/JMST-0161116-2.

[20] P. Bradshaw, Turbulent secondary flows, Annual Review of Fluid Mechanics 19 (1987). 53-74. doi: doi.org/10.1146/annurev.fl.19.010187.000413. 
[21] D. Liu, W. Tang, J. Wang, H. Xue, and K. Wang, Hybrid RANS/LES simulation of sloshing flow in a rectangular tank with and without baffles, Ships and Offshore Structures, 12(8) (2017) 10051015. doi: 10.1080/17445302.2017.1301341.

[22] S. Dahlström and L. Davidson, Hybrid RANS/LES employing interface condition with turbulent structure. In K. Hanjalić, Y. Nagano, \& M.J. Tummers (Eds.), Turbulence, Heat and Mass Transfer 4: Proceedings of the Fourth International Symposium on Turbulence, Heat and Mass Transfer, Antalya, Turkey, 12 - 17 October, 2003 (pp 689-696). New York, NY: Begell House. ISBN: 1567001963.

[23] L. Davidson and S.H. Peng, Hybrid LES-RANS modelling: a one-equation SGS model combined with a $k-\omega$ model for predicting recirculating flows, International Journal for Numerical Methods in Fluids 43 (2003) 1003-1018. dol: 10.1002/d.512.

[24] A.A. Orkomi, K. Ashrafi, and M.S. Motlagh, New plume rise modelling in a turbulent atmosphere via hybrid RANS-LES numerical simulation, Journal of Wind Engineering \& Industrial Aerodynamics 173 (2018) 132-146.

[25] W. Rodi, Comparison of LES and RANS calculations of the flow around bluff bodies, Journal of Wind Engineering and Industrial Aerodynamics 71 (1997) 55-75. doi: doi.org/10.1016/S01676105(97)00147-5.

[26] B. Jaffŕezic, M. Breuer, O. Chikhaoui, G. Deng, and M. Visonneau, Towards hybrid LES-RANScoupling for complex flows with separation. In S. Salmon, E. Sonnendrücker, M. Dumbster, \& C.-S. Munz (Eds.), ESAIM: Proceedings, Vol. 16, CEMRACS 2005 - Computational Aeroacoustics and Computational Fluid Dynamics in Turbulent Flows (2007) (pp. 89-113). London: EDP Sciences. doi: 10.1051/proc:2007010.

[27] W. P. Bennett, A time-accurate analysis of two-dimensional wakes, PhD thesis, University of Leicester, May 2005.

[28] P. L. Roe, Approximate Riemann Solvers, Parameter Vectors, and Difference Schemes, Journal of Computational Physics 43 (1981) 357-372. doi: doi.org/10.1016/0021-9991(81)90128-5. 
[29] B. Van Leer, Towards the Ultimate Conservative Difference Scheme V. A Second-order sequel to Godunov's method, Journal of Computational Physics 32 (1979) 101-136. doi: doi.org/10.1016/0021-9991(79)90145-1.

[30] M. J. Kermani and E. G. Plett, Modified Entropy Correction Formula for the Roe. Paper 20010083, AIAA (2001).

[31] F.Q. Hu, M.Y. Hussain, and J. Manthey, Application of low dissipation and dispersion RungeKutta schemes to benchmark problems in computational aeroacoustics. In: Hardin, J.C., Ristorcelli, J.R., Tam, C.K.W. (Eds.), ICASE/LaRC Workshop Benchmark Problems, in Computational Aeroacoustics (CAA), NASA CP 3300, 24-26 October 1994, Hampton, VA, USA.

[32] European Research Community on Flow, Turbulence and Combustion, ERCOFTAC Classic Collection Database [Dataset] (1999). Retrieved from http://cfd.mace.manchester.ac.uk/ercoftac/.

[33] C. Rumsey, S. Allmaras, J. Alonso, B. Bush, T. Chevalier, A.D. Darian, et al., Recommended Practice: The CFD General Notation System - Standard Interface Data Structures (AIAA Report No. R-101A-2005) (2005), Reston, VA: American Institute of Aeronautics and Astronautics. doi: doi.org/10.2514/4.479304.001.

[34] S. Jakirlić and K. Hanjalić, A second moment closure for non-equilibrium and separating high and low-Re number flows. In F. Durst, B.E. Launder, F. Schmidt, \& J.H. Whitelaw (Eds.), Tenth Symposium on Turbulent Shear Flows (1995) (pp. 23-25). University Park, PA: Pennsylvania State University. 